\title{
Electron emission observations from as-grown and vacuum-coated NDP chemical vapor deposited diamond
}

\author{
A. Lamouri and Yaxin Wang \\ General Vacuum, Inc., Cleveland, Ohio 44105 \\ G. T. Mearini, I. L. Krainsky, and J. A. Dayton, Jr. \\ NASA Lewis Research Center, Cleveland, Ohio 44135 \\ W. Mueller \\ Research 2000, Inc., Westlake, Ohio 44145
}

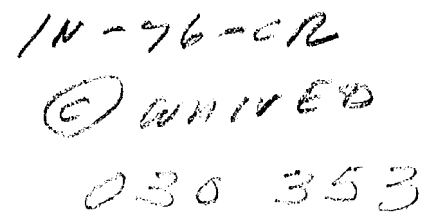

(Received 3 August 1995; accepted 5 February 1996)

\begin{abstract}
Field emission has been observed from chemical vapor deposited diamond grown on Mo and $\mathrm{Si}$ substrates. Emission was observed at fields as low as $20 \mathrm{kV} / \mathrm{cm}$. The samples were tested in the as-grown form, and after coating with thin films of $\mathrm{Au}, \mathrm{CsI}$, and $\mathrm{Ni}$. The emission current was typically maximum at the onset of the applied field, but was unstable, and decreased rapidly with time from the as-grown films. Thin Au layers, $\sim 15 \mathrm{~nm}$ thick, vacuum deposited onto the diamond samples significantly improved the stability of the emission current at values approximately equal to those from uncoated samples at the onset of the applied field. Thin layers of CsI, $\sim 5 \mathrm{~nm}$ thick, were also observed to improve the stability of the emission current but at values less than those from the uncoated samples at the onset of the applied field. While Au and CsI improved the stability of the emission, Ni was observed to have no effect. (C) 1996 American Vacuum Society.
\end{abstract}

\section{INTRODUCTION}

Recently, there has been a growing interest to study the properties of chemical vapor deposited (CVD) diamond for possible application in many electronic devices. In part, this was motivated by the observation of high secondary electron yields and field emission at relatively low fields. Secondary electron emission yields of $\sigma=12-50$ have recently been observed from CVD diamond. ${ }^{1-4}$ These yields are 20 times greater than those from materials presently used in secondary electron emitter applications. ${ }^{5}$ State of the art field emitters generally require applied fields above $5 \times 10^{7} \mathrm{~V} / \mathrm{cm}^{6}$ Room temperature field emission has recently been observed by Shovlin and Kordesch at fields as low as $30 \mathrm{kV} / \mathrm{cm}^{7}$

Unassisted field emission from CVD randomly oriented polycrystalline diamond films grown on $\mathrm{Si}$ and Mo has been demonstrated and observed at room temperature at fields as low as $20 \mathrm{kV} / \mathrm{cm}$. The emission was observed from $1 \times 1 \mathrm{~cm}^{2}$ samples. The applied field for emission ranged from 20 to $160 \mathrm{kV} / \mathrm{cm}$. The total current densities ranged from $1 \mu \mathrm{A}$ to $0.1 \mathrm{~mA} / \mathrm{cm}^{2}$, averaged over the entire sample.

The mechanism responsible for the field emission from CVD diamond at such low fields is not understood at present and is possibly the result of the negative electron affinity of diamond, although Shovlin and Kordesch ${ }^{7}$ speculate that the presence of an adlayer such as gold would quench the surface states responsible for this property of CVD diamond.

In this article we will present cold field emission data from CVD diamond on $\mathrm{Si}$ and $\mathrm{Mo}$ in the as-grown form and after being coated with thin layers of $\mathrm{Au}, \mathrm{CsI}$, and $\mathrm{Ni}$.

\section{EXPERIMENTAL SETUP}

The CVD diamond films were made by depositing a 10-20 $\mu \mathrm{m}$ continuous film on $1 \times 1 \mathrm{~cm}^{2} \mathrm{Si}$ or Mo substrates by thermal decomposition of $1 \%$ methane in hydrogen using a hot tungsten filament technique. The substrates were polished with $1 / 4 \mu \mathrm{m}$ diamond paste and ultrasonically cleaned in alcohol before they were introduced into the deposition chamber. $\mathrm{Au}$ and $\mathrm{Ni}$ were evaporated on the diamond samples from $\mathrm{Al}_{2} \mathrm{O}_{3}$ crucibles and CsI was evaporated from a Ta boat in a diffusion pumped high vacuum chamber that was operated at $3 \times 10^{-8}$ Torr. The thickness of the $\mathrm{Au}, \mathrm{Ni}$, and CsI layers was determined using a quartz crystal microbalance (QCM) which was calibrated with a thickness interferometer. Following the deposition, the substrates were removed from the deposition chamber and immediately introduced into the test chamber.

The field emission tests were performed in an ion pumped high vacuum chamber at a base pressure of $7 \times 10^{-7}$ Torr. The measurements were carried out using an anode with a diameter $(\sim 5 \mathrm{~mm})$ slightly smaller than the dimensions of the samples, which were approximately $1 \mathrm{~cm}^{2}$. This anode was used in order to eliminate possible field emission currents emanating from the "sharp" edges of the cathode. The measurements were obtained by applying up to $16 \mathrm{kV}$ across a $1 \mathrm{~mm}$ gap separating the anode and the emitting cathode as is shown in Fig. 1. The high voltage was applied to the anode and the emission current was measured at the cathode as a function of applied field. The field was varied by increasing the applied voltage or by varying the cathode-anode separation.

\section{RESULTS AND SUMMARY}

After the diamond samples were introduced into the measurement chamber, a gradually increasing field was applied 


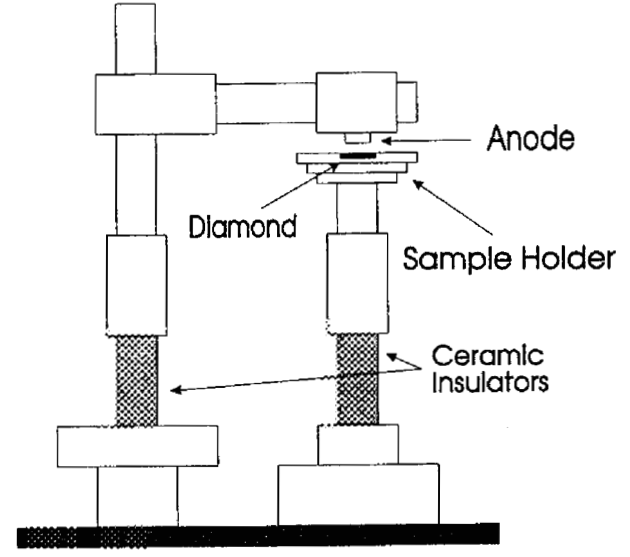

FIG. 1. Schematic diagram of field emission measurements setup.

in order to induce emission. Typical "turn-on" fields for Si and Mo diamond-coated electrodes ranged from 20 to 50 $\mathrm{kV} / \mathrm{cm}$.

For the uncoated samples, the electron emission currents were typically maximum at the onset of the applied field, but were unstable, and decreased rapidly as a function of time. As a result, no meaningful $I-V$ data could be recorded from the as-grown CVD diamond samples.

Relatively stable emission currents with values equal to or close to those from the uncoated samples at the onset of the applied field were observed when the diamond films were coated with thin layers of $\mathrm{Au}$. In addition to a significant improvement in stability, a substantial enhancement of the field emission currents was observed when Au films approximately $15 \mathrm{~nm}$ thick were present on the diamond surface. As shown in Fig. 2, the emission currents from CVD diamond samples on Mo were relatively stable and increased with $\mathrm{Au}$ coverage between 0.5 and $15 \mathrm{~nm}$. For coverages greater than $15 \mathrm{~nm}$, the emission currents, although relatively stable, de-

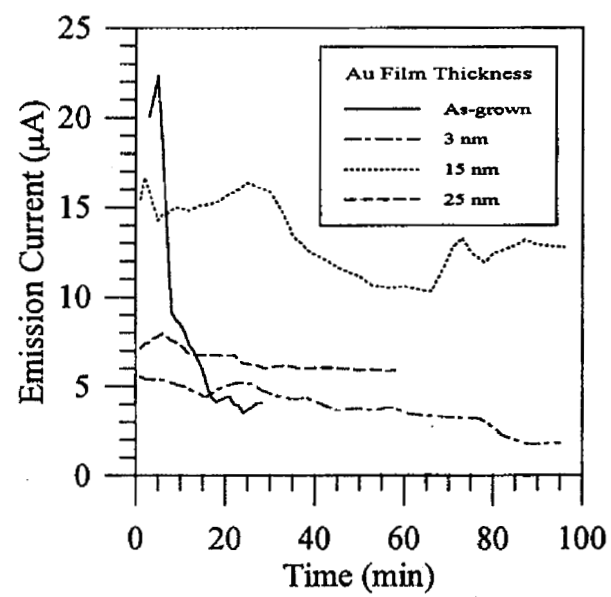

Frg. 2. Time dependence of emission current at $100 \mathrm{kV} / \mathrm{cm}$ from CVD diamond-coated Mo as a function of Au coverage.

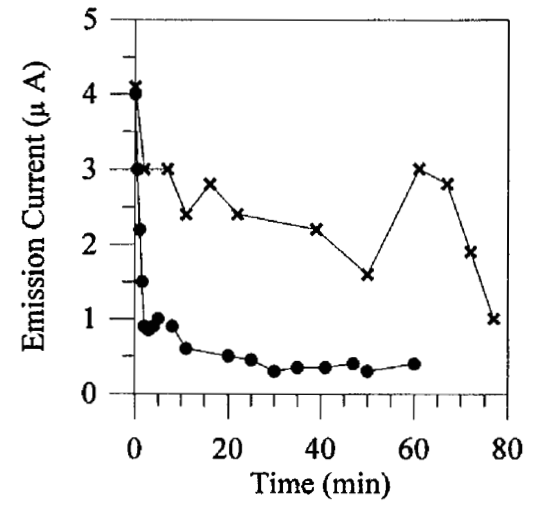

Fig. 3. Emission current at $80 \mathrm{kV} / \mathrm{cm}$ from diamond-coated Si before (dots) and after (crosses) coating with $10 \mathrm{~nm}$ of Au.

creased rapidly as a function of $\mathrm{Au}$ coverage. No emission currents were observed from these films when coated with a thick layer $(\sim 130 \mathrm{~nm})$ of $\mathrm{Au}$.

The data presented in Fig. 2 were obtained from the same CVD sample, that was tested in the as-grown form and with incremental Au coverages. Samples that were tested only after they were coated did not have maximum emission current like those from the as-grown samples at the onset of the applied field. The emission currents from these samples were similar to those of Fig. 2 at comparable Au coverages.

Similar data were obtained for CVD diamond grown on Si substrate, except that the emission currents from diamondcoated Si were significantly less stable before they were coated with Au. As shown in Fig. 3, the emission current from the as-grown samples are very unstable and significantly lower than those from Mo-coated CVD diamond at the onset of applied fields.

Typical data recorded from CVD diamond samples on $\mathrm{Si}$ substrates coated with $10 \mathrm{~nm}$ of $\mathrm{Au}$ are plotted in Fig. 4. These data indicate that, within the experimental field range,

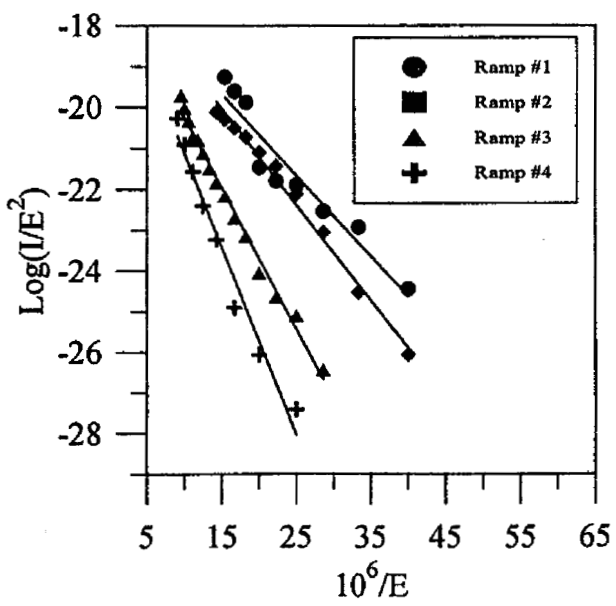

FIG. 4. Fowler-Nordheim plots of emission currents from CVD diamond on Mo coated with $10 \mathrm{~nm}$ of $\mathrm{Au}$. 


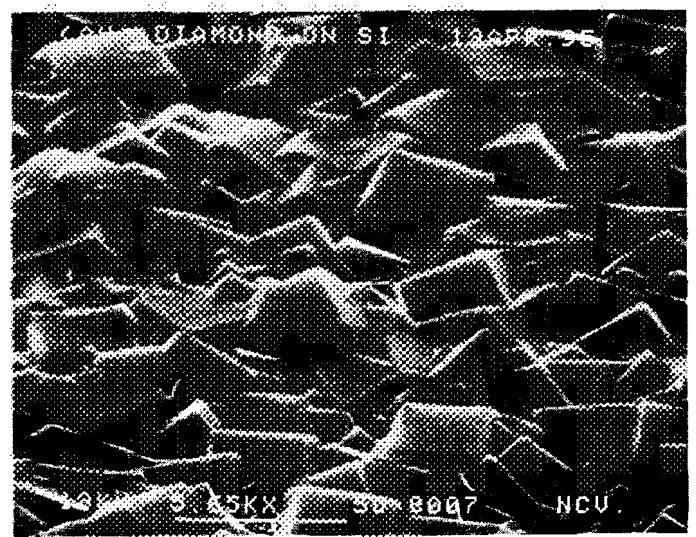

(a)

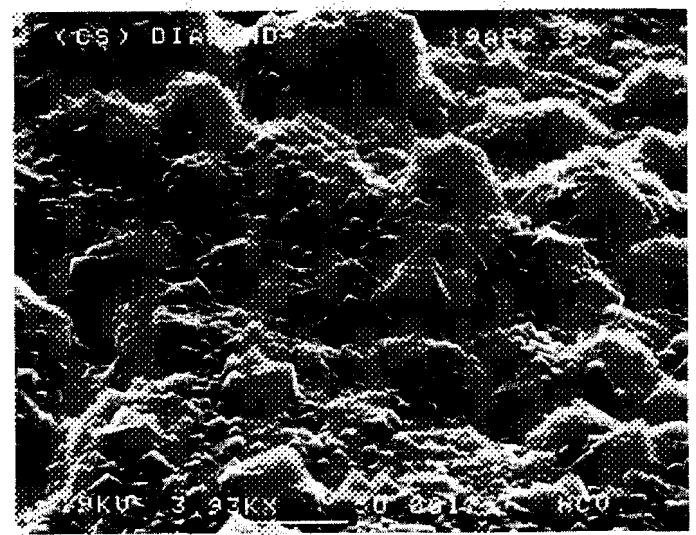

(b)

FIG. 5. SEM photographs showing the surface morphology of CVD diamond films grown on (a) Si and (b) Mo.

the $I-V$ characteristics are well described by the FowlerNordheim regime. In addition, they show that even the Aucoated samples are unstable for high fields since stronger fields are required to obtain the same field emission currents from successive voltage ramps.

Scanning electron microscopy (SEM) analysis of the $\mathrm{Si}$ and Mo coated CVD diamond samples showed that the films had well-faceted structures. Typical SEM photographs from CVD diamond samples on Mo and Si substrates are shown in Figs. 5(a) and 5(b), respectively. Figures 5(a) and 5(b) show that the surface roughness of the Mo-coated CVD diamond is higher (low magnification SEM photographs show that the Si-coated diamond samples are flat over the entire surface area of the samples). It is possible that the surface roughness is at least partially responsible for the higher and more stable electron emission currents from the films grown on Mo.

Our data for Au-coated CVD diamond on Mo and Si are in good agreement with those of Shovlin and Kordesch. ${ }^{7}$ In fact, they were only able to observe field emission current from their CVD diamond films after the samples were coated with $15 \mathrm{~nm}$ of $\mathrm{Au}$, presumably due to the enhancement of emission current at this coverage.

Preliminary data from thin layers of CsI $(0.1$ and $5 \mathrm{~nm})$, vacuum deposited onto the diamond samples, significantly

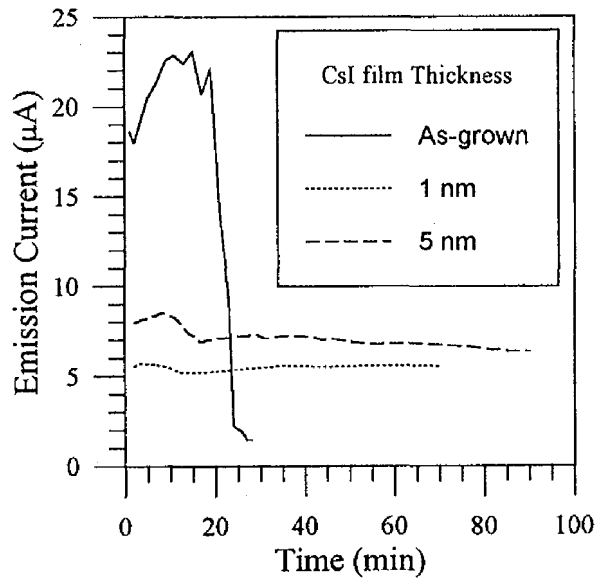

FIG. 6. Time dependence of emission current at $100 \mathrm{kV} / \mathrm{cm}$ from CVD diamond on Mo as a function of CsI coverage.

improved the stability of the emission current at values comparable to those from Au at the same coverage, as is shown in Fig. 6. These results are not surprising since CsI films 5-10 $\mathrm{nm}$ thick are known to stabilize and enhance secondary electron emission (SEE) from CVD diamond after they have been activated by electron bombardment. ${ }^{4}$ Measurement for thicker CsI films on CVD diamond-coated Mo are currently underway and it is possible that more stable and enhanced emission currents will be obtained for coverage of $\sim 10-20$ nm.

The mechanism responsible for the stability of the emission currents induced by CsI and the stability and enhancement induced by $\mathrm{Au}$ is not understood at present. It is possible that hydrogen termination of the surface, believed to be responsible for stabilizing and enhancing SEE from CVD diamond, ${ }^{1,2}$ is stabilized when $\mathrm{Au}$ or CsI is present on the surface. In fact, the instability of the coated samples for long exposures is believe to be the result of sputter erosion of hydrogen due to ions formed close to the cathode, presum-

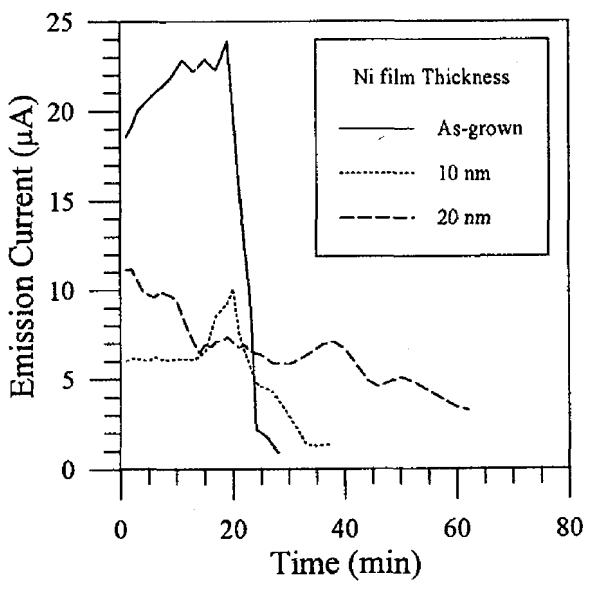

FIG. 7. Time dependence of emission current at $100 \mathrm{kV} / \mathrm{cm}$ from CVD diamond on $\mathrm{Mo}$ as a function of $\mathrm{Ni}$ coverage. 
ably due to the relatively high pressures (mid $10^{-7}$ Torr) in our test chamber. In their measurements of secondary yields from CVD diamond, Mearini et al. ${ }^{1,2}$ discovered that the total secondary yields are significantly reduced when the substrates are bombarded by high-energy hydrogen ions due to graphitization of the diamond surface.

We have also tested field emission from CVD diamond films on Mo coated with Ni. Unlike Au and CsI, Ni does not appear to have a stabilizing effect on the electron emission. As shown in Fig. 7, the emission current for Ni-coated CVD diamond were observed to decrease very rapidly as a function of time, although the maximum emission currents at the onset of the applied field were comparable to those from diamond samples coated with $\mathrm{Au}$ and CsI.

All of the field emission experiments presented here were repeated on bare $\mathrm{Al}, \mathrm{Cu}, \mathrm{Ta}, \mathrm{Mo}$, and Si substrates, and those substrates coated with $\mathrm{Au}, \mathrm{CsI}, \mathrm{Ni}, \mathrm{Ti}$, and $\mathrm{BaF}_{2}$. No emission was observed from these materials with applied field up to $300 \mathrm{kV} / \mathrm{cm}$, well beyond the fields at which stable emission was observed when diamond was included.

\section{ACKNOWLEDGMENTS}

This work was funded in part by SBIR Contract No. NAS3-27369 and The National Research Council.

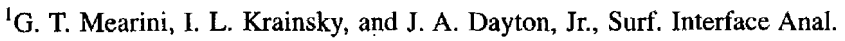
21, 138 (1994).

${ }^{2}$ G. T. Mearini, I. L. Krainsky, Y. X. Wang, J. A. Dayton, Jr., R. Ramesham, and M. F. Rose, Thin Solid Films 253, 151 (1994).

${ }^{3}$ G. T. Mearini, I. L. Krainsky, J. A. Dayton, Jr., Yaxin Wang, Christian A. Zorman, John C. Angus, and R. W. Hoffman, Appl. Phys. Lett. 65, 2702 (1994).

${ }^{4}$ G. T. Mearini, I. L. Krainsky, J. A. Dayton, Jr., Yaxin Wang, Christian A. Zorman, John C. Angus, and D. F. Anderson, Appl. Phys. Lett. 66, 242 (1995).

${ }^{5}$ A. S. Gilmour, Jr., Microwave Tubes (Artech House, Norwood, MA, 1986), pp. 134-137.

${ }^{6}$ I. Brodie and C. A. Spindt, Adv. Electron. Electron Phys. 83, 1 (1992).

${ }^{7}$ J. D. Shovlin and M. E. Kordesch, Appl. Phys. Lett. 65, 863 (1994). 